\title{
Chemical compositions of photometric solar-analog stars and F-G stars of different ages
}

\author{
I. Bikmaev ${ }^{1}$, A. Galeev ${ }^{1}$, N. Sakhibullin ${ }^{1}$ and F. Musaev ${ }^{2}$ \\ ${ }^{1}$ Kazan State University and Academy of Sciences of Tatarstan, Kazan, 420111, Russia \\ email: Ilfan.Bikmaev@ksu.ru \\ ${ }^{2}$ Special Astrophysical Observatory of Russian Academy of Sciences, Karachai-Cherkessian \\ Republic, 369167, Russia \\ email: faig@sao.ru
}

Abstract. We present the main results of our study of photometric solar-analog stars.

Keywords. Stars: abundances, techniques: spectroscopic

\section{Solar-analog stars}

We have obtained spectra of the 15 photometric solar analogs from the list of Kharitonov \& Mironov (1998) by using Coudé-échelle spectrometer (Musaev et al. (1999)) of the 2-m Zeiss reflector at Terskol Observatory. Spectral resolution is $\mathrm{R}=45000$ in a wavelength interval from 4000 to $9000 \AA$, S/N ratio is $150-200$ over the most of the spectral range.

Effective temperatures have been calculated by using published photometric indices and calibrations of Alonso et al. (1996). Surface gravities are based on HIPPARCOS parallaxes. Table 1 gives the obtained atmospheric and physical parameters of the stars. Kurucz (1993) atmosphere models used for abundance pattern determinations. Atomic parameters of spectral lines have been extracted from VALD (Kupka et al. (1999)). To prevent influence of oscillator strengths uncertainties we have used line-by-line approach to determine abundance patterns relative to the solar compositions. Abundance patterns of 33 elements have been obtained for each star and compared with the solar ones. They indicate that photometric analogs of the Sun can be divided into three groups according to their abundances: six stars have solar chemical composition, four have abundance excesses $(\sim 0.2 \mathrm{dex})$, and five have some abundance deficiencies $(\sim 0.2-0.3 \mathrm{dex})$ Galeev et al. (2004). It was found that our sample contains two metal-deficient subgiants (HD 133002 and HD 225239). Figure shows mean abundance pattern differences of 13 photometric analog stars relative to the solar ones.

\section{F-G stars of different ages}

We have constructed a new Coudé-échelle spectrometer for the Russian-Turkish 1.5-m optical telescope (RTT150) installed earlier at TUBITAK National Observatory (Antalya, Turkey), Bikmaev et al. (2005). During 2003-2004 this spectrometer has been tested. The spectrometer consists of two cameras providing nominal resolutions of $\mathrm{R}=$ 40000 and $\mathrm{R}=100000$. Test observations have shown high enough positional and spectrophotometric accuracies of RTT150 CES $(0.1 \mathrm{~km} / \mathrm{s}$ for radial velocity and $2-4 \mathrm{~m} \AA$ for equivalent widths accuracies). In 2004 we have started at RTT150 with spectroscopic investigation of the sample of F-G stars of different ages from the list of Nordstrom et al. 
Table 1. Main parameters of photometric solar analogs.

\begin{tabular}{l|c|c|c|c|c|}
\hline HD & $T_{\text {eff }} \mid$ & $\lg g$ & {$[\mathrm{Fe} / \mathrm{H}]$} & Mass & Age \\
\hline 159222 & 5805 & 4.39 & 0.13 & 1.00 & 6.0 \\
186408 & 5740 & 4.24 & 0.10 & 1.00 & 9.4 \\
222143 & 5720 & 4.41 & 0.10 & 0.95 & 10.4 \\
34411 & 5800 & 4.20 & 0.08 & 1.00 & 9.2 \\
10307 & 5815 & 4.32 & 0.05 & 1.00 & 7.7 \\
141004 & 5870 & 4.18 & 0.05 & 1.05 & 8.3 \\
186427 & 5700 & 4.34 & 0.05 & 0.95 & 9.4 \\
213575 & 5630 & 4.15 & 0.05 & 0.95 & 10.3 \\
146233 & 5710 & 4.37 & -0.01 & 0.95 & 4.6 \\
197076 & 5810 & 4.46 & -0.05 & 1.05 & 5.2 \\
187923 & 5700 & 4.08 & -0.12 & 1.02 & 10.4 \\
4307 & 5780 & 3.98 & -0.17 & 1.10 & 7.2 \\
4915 & 5660 & 4.59 & -0.24 & 0.90 & 12.8 \\
133002 & 5610 & 3.45 & -0.38 & 1.53 & 1.7 \\
225239 & 5650 & 3.79 & -0.39 & 1.30 & 5.8 \\
\hline Mean of & 5750 & 4.28 & 0.0 & 1.00 & 8.0 \\
13 stars & \pm 70 & \pm 0.10 & \pm 0.11 & \pm 0.05 & \pm 2.5 \\
\hline
\end{tabular}

(2004). Up to date we have obtained spectra of 30-40 stars in the age range of 1-15 Gyr. This work is in progress and we are planning to obtain abundance patterns of the elements for these stars using the same approach as for the solar-analog stars.

\section{Acknowledgements}

These studies have supported by grants of RFBR 05-02-17744 and Leading Schools NSh-1789.2003.2. Authors are grateful to Kazan State University, Academy of Sciences of Tatarstan (Russia), and TUBITAK National Observatory (Turkey) for financial supports of RTT150 Coude-echelle spectrometer construction.

\section{References}

Alonso A., Arribas S. \& Martinez-Roger 1996, Astron. Astrophys. 313, 873

Bikmaev I.F., Sakhibullin N.A., Musaev F.A., \& Aslan Z. 2005, in preparation.

Galeev A.I., Bikmaev I.F., Musaev F.A., \& Galazutdinov G.A. 2004, Astronomy Reports 48, 492

Kharitonov A.V. \& Mironov A.V. 1998, in: Hill J. (ed.), Second Annual Lowell Observatory Workshop, Flagstaff, Arizona, October 5-7, 1997, 149

Kupka F., Piskunov N., Ryabchikova T.A., et al. 1999, Astron. Astrophys. Suppl. Ser 138, 119

Kurucz R.L. 1993, Grid of Stellar Atmospheres on CD-ROMs (Cambridge)

Musaev F.A., Galazutdinov G.A., Sergeev A.V., Karpov N.V., \& Pod'yachev Yu.V. 1999, Kinematics and Physics of Celestial Bodies 13, 282

Nordstrom B., Mayor M., Andersen J., et al. 2004, Astron. Astrophys. 418, 989 